\title{
Sol-gel Synthetic Route to Improve Interaction of Polyester/Cotton Blended Fabric with Anionic Dyes
}

\author{
Tarek Salem ${ }^{1 *}$, Madiha El-Kashouty ${ }^{1}$, Martin Müller², Frank Simon ${ }^{2}$ \\ ${ }^{1}$ Textile Research Division, National Research Centre El-Buhoose St., 12622, Cairo, \\ Egypt. ${ }^{2}$ Leibniz Institute of Polymer Research Dresden, Hohe Str. 6, D-01069 Dresden, \\ Germany.
}

\begin{abstract}
$\mathbf{T}$ HE POTENTIAL of PET/cotton fabric blends is reflected by a wide spectrum of applications including domestic and technical textiles. However, a different chemical nature of their fibers hinders to attain solid shade during the coloration process. To overcome this problem in current attempt, surface functionalization of PET/cotton fabric was investigated. This can be reached by simple and cost effective sol-gel technology. By this simple coating method different anchor sites were introduced onto the PET/cotton fabric surface such as amine, epoxy or hydroxyl. Incorporation of such groups was conducted using two different alkoxysilanes, namely 3-Aminopropyltriethoxysilane (APTES) or 3-Glycidyloxypropyltrimethoxysilane (GPTMS) as coupling agents for C.I. reactive blue 5 and C.I. acid blue 80. The sol-gel synthesis reactions of used alkoxysilanes were preceded under either acidic or basic condition. The chemistry and morphology of APTES and GPTMS modified PET/cotton surface were studied by utilizing some surface sensitive techniques such as streaming potential measurements, ATRFTIR, X-ray photoelectron spectroscopy (XPS), time-dependent contact angle measurements as well as scanning electron microscopy (SEM). In addition, color strength and fastness tests of the prints were done to test the effectiveness of the fabric surface modification. The results presented that the PET/cotton blend fabrics after treatment with base hydrolyzed APTES showed improvement in $\mathrm{K} / \mathrm{S}$ value and fastness properties.
\end{abstract}

Keywords: Sol-gel coating, Silanes, X-ray photoelectron spectroscopy (XPS),3Aminopropyltriethoxysilane, Blended fabrics.

\section{Introduction}

The functionalization or modification of fabric surfaces via anchoring polymer layers plays a central role in controlling the surface charge and properties. Among all types of fabrics, blended fabrics are particularly interesting as they exhibited the combination of remarkable attributes of both blended fibers [1]. They allow the production of fabrics having good wear properties, dimensional stability, mechanical strength, abrasion resistance and attractive handle. However, because different fibers have a different chemical structure, blends suffer from inherent disadvantages [2]. For example, coloration requires different classes of dyestuffs during the two-stage process to produce solid shades. This significantly hinders the concurrent coloration process [3]. Therefore, immobilization of coating layers on blended surfaces could be a promising approach for varieties of applications, and reduce complexity in effluent treatment [4]. For this purpose, surface functionalization of textile blends has been studied in order to control its properties.

The sol-gel technology has been widely applied for the preparation and application of coatings based on either inorganic materials or inorganic-organic hybrids [5]. It is a wet process that can be accomplished with techniques commonly used in the textile industry [6]. At the same time, it gives the chance to adapt desired properties in a single coating step [7]. This synthetic route involves the phase transition of a metal-organic precursor from a liquid "sol" into a solid "gel" phase. The "gel" is converted into coating thin films with further drying and heat-treatment [8-10]. The sol-gel technology can be applied to modify surface properties of 
various types of fibrous materials. For example, it is possible to increase the hydrophobicity, oleophobicity and soil repellency of fibers by coating them with silica nanosols containing perfluoroalkyl compounds [11, 12]. Chen et al. reported an approach for manufacturing environmentally robust superhydrophilic wool fabrics by coating an ultrathin silica film onto wool fabrics [13]. By using modified silica sol, Gao et al. developed highly hydrophobic surfaces on polyester and cotton fabrics. Such modifying system formed by hydrolysis and subsequent condensation of tetraethoxysilane under basic condition. Afterward, hydrolyzed hexadecyltrimethoxysilane (HDTMS) was used to hydrophobize silica sol [14]. UVabsorbers such as $\mathrm{ZnO}$ or $\mathrm{TiO}_{2}$ embedded into nanosol coatings can be applied to prepare UVprotective coatings on fabrics $[15,16]$.

Polyester/cotton blend textiles were developed over 40 years ago [17]. Approximately, 12 million tons of PET was utilized to manufacture staple fibers which were mostly used to blend with cotton fibers to produce PET/cotton blend fabrics [18]. The popularity of these blends in the clothing industry originates from complementary properties of fibers. Polyester/cotton blends have the advantage of polyester's tensile strength, abrasion resistance, and dimensional stability as well as cotton's reduced pilling, ability to absorb water, and comfort in wear [19]. However, the existence of both components polyester and cotton in textiles causes some difficulties in the coloration process. Because of absence reactive chemical groups, polyester's macromolecules display a hydrophobic character. Therefore, it is difficult to apply the majority of dyestuffs apart from disperse dyes, whilst the cotton part of the blend is frequently colored using water soluble reactive dyes. Consequently, this may originate a barrier in simultaneous coloration process of $\mathrm{PET} /$ cotton fabrics [3].

To counter this problem, several approaches have been used to improve PET/cotton blends coloration using a single class of colorant with the aim to enhance the concurrent process of coloration and reduce complexity in effluent treatment. Öktem et al. investigated the coloration of PET/cotton blends with a basic dye by in situ plasma polymerization of acrylic acid [20]. Youssef et al. explored the viability of using a commercially available sodium edetate for alkaline dyeing of polyester and its cotton blend [19]. Ristić et al. studied the effects of two-step process based on using corona discharge on the dyeability of polyester/cotton blended fabric with direct and reactive dyes [21]. Hinks et al synthesized water soluble fiber-reactive dye as a universal dye class for printing on cotton, polyester and polyster/ cotton blend fabrics [22].

This study aimed at constructing a top layer on the outermost surface of PET/cotton blends to improve adhesion between its surface and anionic dyes, such as C.I. Acid Blue 80 and C.I. Reactive Blue 5 (Fig.1) to attain solid shades on PET/cotton blends using one class of dyestuffs. The surface of PET/cotton blends was modified using two different silane coupling agents, namely 3-Aminopropyltriethoxysilane and 3-Glycidyloxypropyltrimethoxysilane (Fig.2) via sol-gel route. As a result, coloration properties became dependent on the properties of the adhered layer rather than chemical composition of the single fibers. This is considered as a key step towards the substrate independent surface coloration.

The effect of modifications on the surface properties of the PET/cotton blends, were assessed by means of a combination of various surface-sensitive characterization methods including XPS, dynamic wetting measurements, and stream potential measurements. In addition, the influence of the functionalization of PET/cotton was investigated via conventional printing by using C.I. Acid Blue 80 and C.I. Reactive Blue 5 dyes and the obtained color strength $(\mathrm{K} / \mathrm{S}$ value) examined by spectrophotometer and the colorfastness properties were also evaluated.

\section{Experimental}

\section{Materials}

Commercial PET/cotton weave blend fabric; with a blend composition of $65 \%$ PET and 35 $\%$ cotton was supplied by El-Mahalla El-Kubra Co., Egypt. The fabric was washed by treatment in a bath containing $0.5 \mathrm{~g} / \mathrm{L}$ non-ionic detergent and sodium carbonate $1 \mathrm{~g} / \mathrm{L}$ at the liquor-tofabric ratio of $50: 1$ for $30 \mathrm{~min}$ at $50{ }^{\circ} \mathrm{C}$ followed by rinsing with deionized water, dried at room temperature until constant weight and kept in desiccators before use in the experiments. 3-Aminopropyltriethoxysilane (APTES, Acros), 


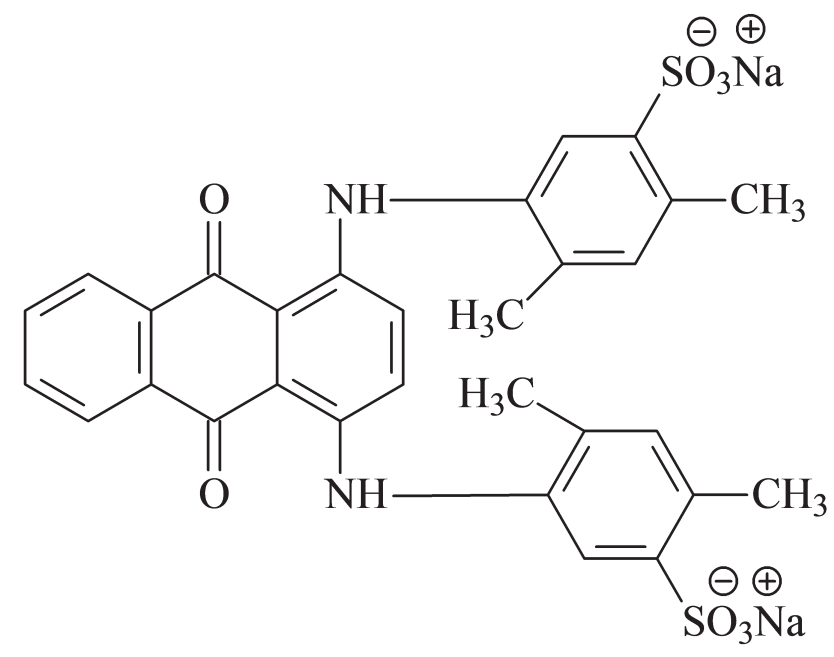

C.I. Acid Blue 80<smiles>Nc1c(S(=O)(=O)O)cc(Nc2ccc(S(=O)(=O)O)c(Nc3nc(Cl)nc(Nc4cccc(S(=O)(=O)O)c4)n3)c2)c2c1C(=O)c1ccccc1C2=O</smiles>

C.I. Reactive Blue 5

Fig. 1. Dye structure of (a) C.I. Acid Blue 80 and (b) C.I. Reactive Blue 5.<smiles>CCO[Si](CCCN)(OCC)OCC</smiles>

APTES<smiles>CO[Si](CCCOCC1CO1)(OC)OC</smiles>

GPTMS

Fig.2. Surface-modifying alkoxysilanes employed for PET/cotton fabric functionalization.

3-Glycidyloxypropyltrimethoxy-silane (GPTMS, Acros), ammonium hydroxide solution $\left(\mathrm{NH}_{4} \mathrm{OH} ; \quad 28-30 \% \quad\right.$ wt. $\left.\%\right)$, urea, sodium alginate, sodium chlorate, ammonium sulphate, hydrochloric acid, and formic acid (85\%) (El Nasr pharmaceutical chemicals Co.), C.I. Acid Blue 80 and C.I. Reactive Blue 5 dyes (Bruno Ludewig $\mathrm{GmbH}$ ) were used.

Preparation of silica solutions and coating of PET/cotton blended fabric
Silica sols were prepared by using 3-propylaminotriethoxysilane (APTES) or 3-Glycidyloxypropyltrimethoxysilane (GPTMS) as precursors, ammonium hydroxide $\left(\mathrm{NH}_{4} \mathrm{OH}\right.$; 28-30 wt.\%) or hydrochloric acid $(0.01 \mathrm{~N} \mathrm{HCl})$ as catalysts, ethanol as solvent, and distilled water. For silica sols preparation, $2 \mathrm{ml}$ of precursor is stirred into a mixture of $20 \mathrm{ml}$ ethanol and $10 \mathrm{ml}$ distilled water. Afterwards, the hydrolysis and condensation reactions occurred by vigorously stirring overnight at 
room temperature. In acid hydrolysis, $0.01 \mathrm{~N} \mathrm{HCl}$ was used to adjust the $\mathrm{pH}$ of silica sols solution to $\mathrm{pH} 3-4$, while in case of base hydrolysis $\mathrm{pH}$ 10-11 was adjusted with $\mathrm{NH}_{4} \mathrm{OH}$. The silica sols were coated onto PET/cotton blend fabrics by the immersion process at room temperature for 10 min under atmospheric conditions, a wet chemistry method. After the PET/cotton blend fabrics were immersed in the $\mathrm{pH}$-adjusted silica sol solution, the blend samples are air dried at room temperature and afterwards a thermal treatment in a convection oven at 120 ${ }^{\circ} \mathrm{C}$ for $30 \mathrm{~min}$ was performed. Then, modified samples obtained were thoroughly washed with distilled water in order to remove weakly adsorbed APTES or GPTMS.

\section{Instrumentations}

$X$-ray photoelectron spectroscopy (XPS)

XPS measurements were carried out by means of an Axis Ultra X-ray photoelectron spectrometer (Kratos Analytical, Manchester, UK). The spectrometer was equipped with a monochromatic Al K (hv $=1486.6 \mathrm{eV})$ X-ray source of $300 \mathrm{~W}$ at $15 \mathrm{kV}$. The kinetic energy of the photoelectrons was determined with a hemispherical analyser set to pass energy of $160 \mathrm{eV}$ for wide-scan spectra and $20 \mathrm{eV}$ for the high-resolution element spectra. During all measurements, electrostatic charging of the sample was avoided by means of a lowenergy electron source working in combination with a magnetic immersion lens. Later, all recorded peaks were shifted by the same amount that was necessary to set the $\mathrm{C} 1 \mathrm{~s}$ peak to 284.76 $\mathrm{eV}$ for the carbon of the PET's phenyl ring [23]. Quantitative elemental compositions were determined from peak areas using experimentally determined sensitivity factors and the spectrometer transmission function. Spectrum background was subtracted according to Shirley [24]. The highresolution spectra were deconvoluted by means of the spectra deconvolution software (Kratos Analytical, Manchester, UK). Free parameters of component peaks were BE, height, full width at half-maximum, and Gaussian-Lorentzian ratio.

\section{ATR- FTIR Spectroscopy}

Samples of the unmodified and modified PET/cotton blends were analysed by ATR-FTIR spectroscopy using a commercial ATR-FTIR attachment (OPTISPEC, Zürich, Switzerland). The ATR-FTIR attachment was installed on the IFS 55 Equinox FTIR spectrometer (BRUKER Optics $\mathrm{GmbH}$, Ettlingen) equipped with globar source and MCT detector. Samples $\left(2 \times 4 \mathrm{~cm}^{2}\right)$ of the unmodified and different modified PET/cotton blends were pressed against trapezoidal $\mathrm{ZnSe}$ internal reflection elements (IRE) $(50 \times 20 \times 2$ $\mathrm{mm}^{3}$ ). The IRE and blend were clamped together onto a home built in-situ-ATR-FTIR cell. Spectra were recorded at $2 \mathrm{~cm}^{-1}$ resolution between 4000 $\mathrm{cm}^{-1}$ and $400 \mathrm{~cm}^{-1}$.

\section{Streaming potential measurements}

Electrokinetic measurements were carried out to determine the electrokinetic potential $(\zeta)$ of the PET/cotton fabric surface as a function of the $\mathrm{pH}$ values of the electrolyte solution using the streaming potential method. Streaming potential is generated when a liquid is forced to flow directly through a channel formed between two sample surfaces separated by a narrow slit. The streaming potential was determined using EKA electrokinetic analyser from Anton Paar (Graz, Austria). The $\mathrm{pH}$ values were adjusted with 0.1 $\mathrm{mol} \cdot \mathrm{L}^{-1} \mathrm{HCl}$ or $0.1 \mathrm{~mol} \cdot \mathrm{L}^{-1} \mathrm{KOH}$. To prevent a significant change in the ionic strength by adding $\mathrm{HCl}$ and $\mathrm{KOH}$, all measurements were carried out in $10^{-3} \mathrm{~mol} \cdot \mathrm{L}^{-1} \mathrm{KCl}$, which is known as an inert electrolyte. The zeta-potential values were calculated from the measured streaming potentials by the use of the Smoluchowski equation [25].

\section{Kinetics measurements of wetting}

The wetting properties of the PET/cotton fabric surfaces before and after surface functionalization were estimated by means of dynamic wetting measurements, which were carried out with a FibroDAT 1122HS dynamic contact angle tester (Fibro System, Sweden) equipped with a highspeed video camera according to the sessile drop method. A microliter syringe was used to place a deionized water droplet (Millipore water having a surface tension of $\gamma_{\mathrm{lv}}=72.8 \mathrm{mN} / \mathrm{m}$ at $23{ }^{\circ} \mathrm{C}$ ) of a $10 \mu \mathrm{l}$ volume on the fabric surface under investigation by a short stroke from an electromagnet [26]. The strength of stroke was minimized to avoid oscillation effects. The data were collected after the water droplet stabilized on the surface. The instrument was located in a temperature-controlled laboratory maintained at $23 \pm 1^{\circ} \mathrm{C}$. The initial contact angle of water and the absorption time (i.e. time recorded until the drop had completely penetrated into the PET/ cotton fabric surface) were used to evaluate the wettability of PET/cotton fabrics before and after sol-gel treatment. The contact angle and the absorption time of water droplets shown for each sample are averages of five single measurements 
[26].

Scanning electron microscopy (SEM)

Surface morphologies of unmodified and different modified PET/cotton blend fabric surfaces were evaluated by SEM Ultra 55 equipped with field emission gun (Carl Zeiss NTS GmbH, Oberkochen, Germany) operating at electron energy of $2 \mathrm{keV}$. Prior to the analysis, the samples were sputter-coated with a thin layer $3 \mathrm{~nm}$ of platinum under vacuum in an argon atmosphere using a sputter coater (Bal-Tec SCD 500, USA) to avoid or minimize sample charging under the electron beam.

\section{Printing procedure}

Preparation of printing pastes

The printing paste with C.I. acid blue 80 was formulated according to the following recipe:

\begin{tabular}{ll} 
Ingredients & By weight $(\mathbf{\%})$ \\
\hline Dye & 2 \\
Sodium alginate & 5 \\
Urea & 5 \\
Sodium chlorate & 1.5 \\
Ammonium sulphate & 6 \\
Water & 80.5
\end{tabular}

The printing paste with C.I. reactive blue 5 was prepared according to the following recipe:

\begin{tabular}{ll} 
Ingredients & By weight $(\mathbf{\%})$ \\
\hline Dye & 2 \\
Sodium alginate & 5 \\
Urea & 5 \\
Sodium chlorate & 1.5 \\
Formic acid & 1 \\
Water & 85.5
\end{tabular}

\section{Printing and post-treatment methods}

The aforementioned formulated printing pastes of the anionic dyes were applied to the unmodified and different modified PET/ cotton blend fabrics by using a conventional flat screen-printing method [27]. After printing, the printed fabric samples were dried in the air followed by steaming at $102{ }^{\circ} \mathrm{C}$ for $20 \mathrm{~min}$. Then, the printed fabric samples were washed with cold water and soaped using $3 \mathrm{~g} / \mathrm{L}$ of non-ionic detergent at $60{ }^{\circ} \mathrm{C}$ for $15 \mathrm{~min}$ at a liquor-to-fabric ratio of 50:1. Afterward, the samples were rinsed in cold water to remove unfixed dyes and finally air-dried.

\section{fabrics}

Colour strength measurements of printed

The PET/cotton blended fabrics printed under different conditions of treatments were individually tested for their colour strength $(\mathrm{K} / \mathrm{S})$. The K/S values are a function of the spectral reflectance at a given wavelength and are defined as the ratio between light absorption $(\mathrm{K})$ and scattering (S) characteristics of the sample. The colour strength $\mathrm{K} / \mathrm{S}$ values of the printed fabrics were instrumentally determined from reflectance measurements with the following Kubelka Munk equation $[28,29]$.

$$
\frac{\mathrm{K}}{\mathrm{S}}=\frac{(1-\beta)^{2}}{2 \beta} \text { (1) }
$$

Where $(\beta)$ is the degree of remission = $R / 100$. Reflectance $(R)$ obtained from a software calculation is expressed as a percent value. $(\mathrm{S})$ is the scattering coefficient and $(\mathrm{K})$ is the absorption coefficient of the printed fabrics. Therefore, the increase of the $\mathrm{K} / \mathrm{S}$ value can be used as a characteristic for different surface modification approaches applied to PET/cotton blended fabric.

The colorimetric measurement by reflectance was carried out in data color SF plus colorimeter. In the process, the unprinted fabrics were used as a reference. Each fabric sample was folded twice, and three scans were performed at different places on the fabric surface. The average value was recorded.

\section{Colour fastness properties}

The color fastness properties of the printed fabrics to washing and rubbing were assessed by DIN EN 20105-C01:1992 and DIN EN ISO 105X12:2002, respectively.

\section{Results and Discussion}

The current work was undertaken to establish a method to overcome coloration challenges of $\mathrm{PET} /$ cotton blended fabrics by applying sol-gel technology, which offers effective way to prepare stable, transparent, and host matrices for one dyeclass. Toward this goal, the PET/cotton blended fabrics were coated either by APTES or GPTMS. A particular advantage is that the coating system was carried out at normal pressure and room temperature. Moreover, we studied the influence of acid or base catalysts on the properties of the anchored material. Finally, the effectiveness of surface functionalization of PET/cotton blended fabrics and control its surface properties was demonstrated in terms of $\mathrm{K} / \mathrm{S}$ values obtained from the printing experiments with different 
classes of anionic dyes.

\section{Electrokinetic measurements}

Coating of PET/cotton fabric with APTES

The change of the interfacial charge at the PET/cotton surface after APTES coating by so-gel approach was observed using streaming potential measurements. Fig. 3 shows the zeta-potential vs. $\mathrm{pH}$ profiles for PET/cotton fabric surface modified with APTES after either acid or base hydrolysis. Regardless of the type of hydrolysis, in comparison to the unmodified PET/cotton fabric surface, PET/cotton fabric surface covered with APTES shows a shift of the isoelectric point $\left(\mathrm{IEP}=\left.\mathrm{pH}\right|_{\zeta=0}\right)$ toward the basic direction indicating that a significant amount of APTES was bound to the surface. The shifts of IEP toward a higher $\mathrm{pH}$ reveal alterations of chemical composition of PET/cotton surface. It was suggested that these changes caused by an additional contribution of Brønsted basic groups into the surface region of PET/cotton fabric. Moreover, it was observed that the IEP value of PET/cotton fabric coated with base-hydrolyzed APTES is shifted toward higher $\mathrm{pH} 5.5$ in comparison with $\mathrm{PET} /$ cotton fabric coated with acid-hydrolyzed APTES $\mathrm{pH}$ 4.5. Therefore, according to Stern theory [30], the number of Brønsted basic groups on the basehydrolyzed APTES covered PET/cotton fabric is higher than on the acid-hydrolyzed APTES covered PET/cotton fabric. In case of basehydrolyzed APTES covered PET/cotton fabric, the increase in Brønsted basic groups may be ascribed to the type of structure formed on the fabric under acidic or basic conditions employed in the Stöber process. It is assumed that, under basic condition highly branched clusters are formed, which results in large surface area and increase in Brønsted basic groups.

Adsorption of the APTES onto PET/cotton fabric surface arises through ion-dipole interaction may be expected between the positively charged

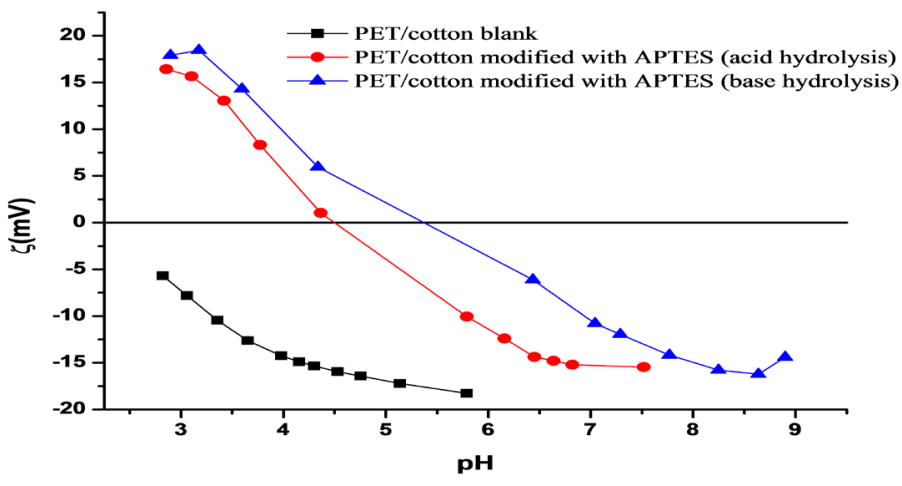

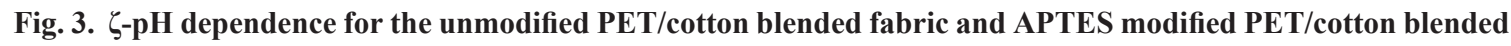
fabric under acid or base hydrolysis.

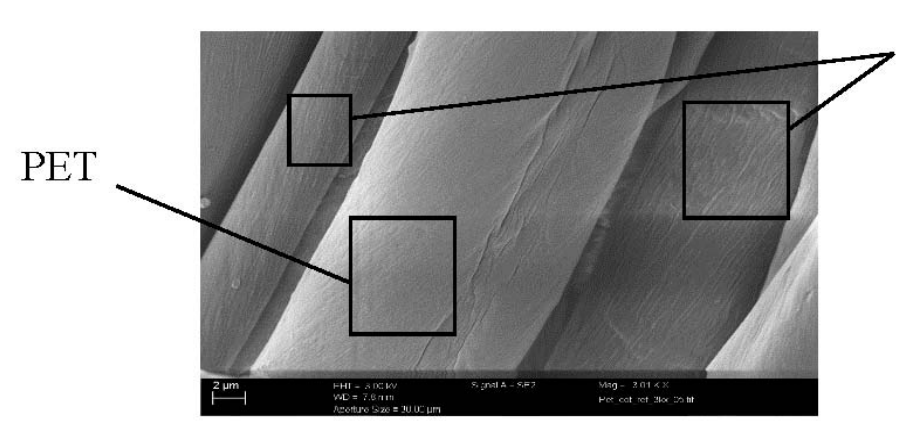

Cotton

Fig. 4. SEM image of PET and cotton fibers in unmodified PET/cotton blended fabric.

Egypt. J. Chem. 60, No.6 (2017) 
nitrogen atom of the APTES and the hydroxyl groups on cellulosic part of blended fabric i.e., cotton fiber provides anchor sites needed for APTES deposition as shown in Fig.4.

Coating of PET/cotton fabric with GPTMS

Figure 5 shows the zeta potential curves exhibited by unmodified and GPTMS modified PET/cotton fabrics under acidic or basic conditions. It was noted that, displacement of acid-hydrolyzed GPTMS PET/cotton curve is not as great as might be expected after changing the surface chemistry. While base-hydrolyzed GPTMS PET/cotton curve displaced towards higher IEP value $(\mathrm{pH}=4)$ if compared with unmodified PET/cotton curve.

As mentioned before, the absolute value of IEP describes the chemical properties of the surface. For this reason, it was assumed that hydroxyl groups on the surface of cotton fiber decreased after epoxysilane reaction as depicted in Fig. 6. The rate trends of assumed reactions can change with reaction conditions. The results indicate that epoxysilane was attached successfully to the surface of the PET/cotton blended fabric under basic conditions. It can be concluded that, the chemical properties of the produced coating

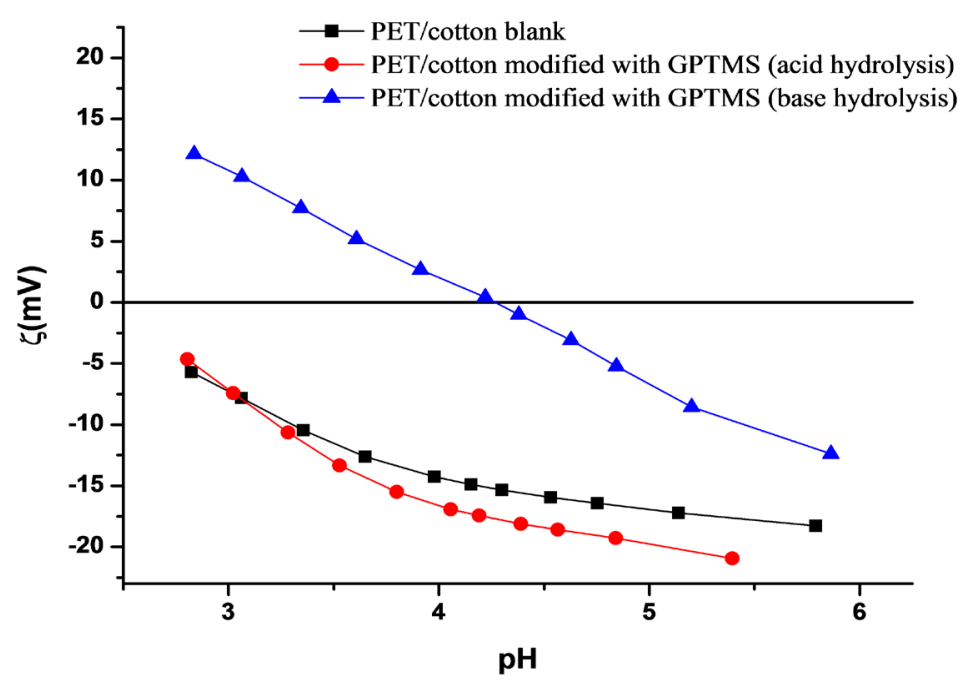

Fig. 5. ร-pH dependence for the unmodified PET/cotton blended fabric and GPTMS modified PET/cotton blended fabric under acid or base hydrolysis.

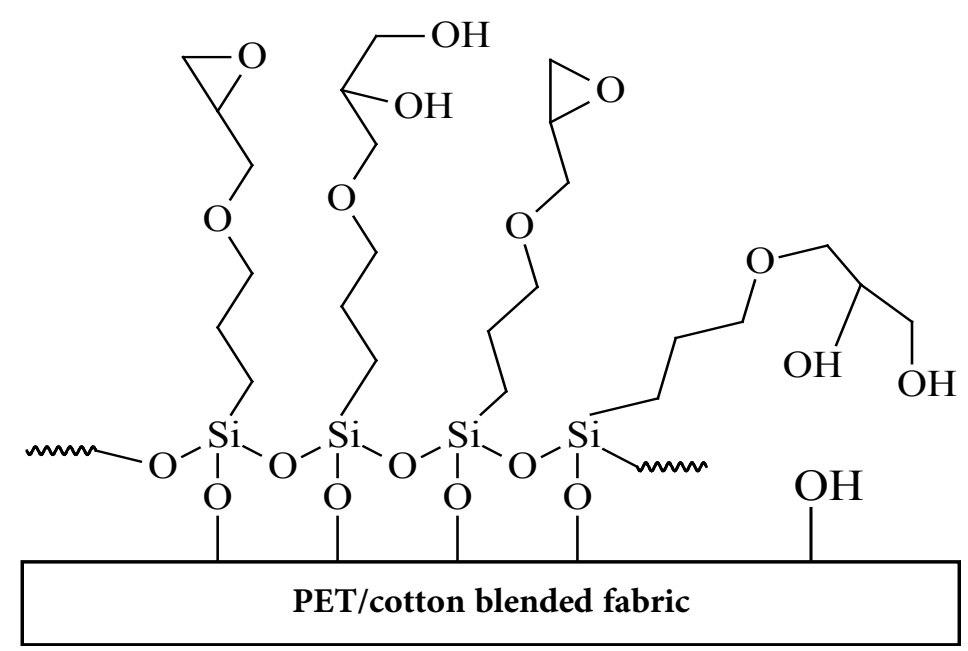

Fig. 6. Schematic representation of attachment GPTMS on PET/cotton fabric surface

Egypt. J. Chem. 60, No.6 (2017) 
depend largely on the type of catalyst used. Moreover, in case of different silanes (APTES, amino silane) and (GPTMS, epoxy silane) we observed the expected difference in the IEP. It seems that the silanes control the surface properties of the fibers.

\section{ATR-FTIR analysis}

FTIR measurements have been done for selected samples to follow the changes in the functional groups that could have occurred as a result of a sol-gel surface modification. Fig. 7 shows the FTIR of the unmodified and modified PET/cotton fabric with two different base-hydrolyzed surfacemodifying alkoxysilanes APTES and GPTMS. The spectrum of the unmodified PET/cotton fabric was very complex because it included absorption bands characteristics of both cellulose and polyester. It was observed that, the FTIR spectrum of either the base-hydrolysed APTES or base-hydrolysed GPTMS did not reveal remarkable changes when compared to the unmodified PET/cotton fabric such as $\mathrm{Si}-\mathrm{O}-\mathrm{Si}$ and $\mathrm{Si}-\mathrm{OH}$ bonds. Although, the streaming potential measurements showed the introduction of new functionalities onto PET/ cotton fabric surface by sol-gel treatment. This

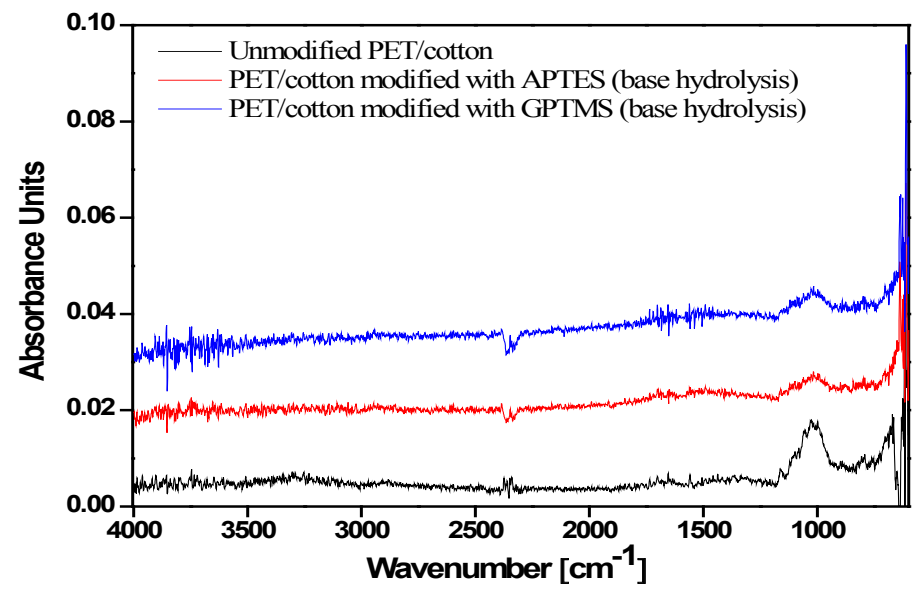

Fig. 7. ATR-FTIR spectra of the unmodified and modified PET/cotton fabric with base hydrolyzed surfacemodifying alkoxysilanes APTES and GPTMS.

can be ascribed to the surface chemical changes resulting from sol-gel modification are confined to a very thin surface layer. Hence, the concentration of the formed new functional groups in this coating layer is not high enough to be measured by ATRFTIR spectroscopy.

Therefore, further investigation was carried out using another sensitive technique XPS to point out and clarify the chemical changes occurred during sol-gel coating process.

\section{XPS characterization}

To investigate the chemical structure of outermost surface, Fig. 8 shows wide-scan XPS spectra of the unmodified PET/cotton and base hydrolyzed APTES modified PET/cotton fabrics. XPS widescan spectrum of the unmodified PET/cotton fabric surface (Fig. 8a) shows two peaks characteristic of carbon $(\mathrm{C} 1 \mathrm{~s})$ and oxygen (O 1s). However, after sol-gel treatment with base hydrolyzed APTES (Fig.8b), one additional peak appeared significantly for nitrogen $(\mathrm{N}$ 1s). The $\mathrm{N}$ 1s peak indicates that the nitrogen-containing functional groups were Egypt. J. Chem. 60, No.6 (2017) incorporated onto the surface of the PET/cotton fabric by sol-gel treatment.

The elemental surface compositions of surface region and the corresponding atomic ratios of the unmodified and base hydrolyzed APTES modified PET/cotton fabrics are summarized in Table 1. Table 1 demonstrates that after the sol-gel treatment with base hydrolyzed APTES the nitrogen content increases, and consequently the $[\mathrm{N}]:[\mathrm{C}]$ atomic ratio increases. The increase in nitrogen content confirms the increase in the number of nitrogen functionalities after sol-gel treatment.

Additional proof into the surface chemistry can be gained through deconvolution of the XPS spectra to examine what were chemical functional groups introduced onto the surface of the PET/cotton fabric after sol-gel treatment with base hydrolyzed APTES. The high-resolution N 1s spectrum (Fig. 9) confirmed the addition of different nitrogen functionalities to the PET/cotton surface. The highresolution $\mathrm{N}$ 1s spectrum was decomposed into two main component peaks (component peaks L and $\mathrm{M}$ ) 


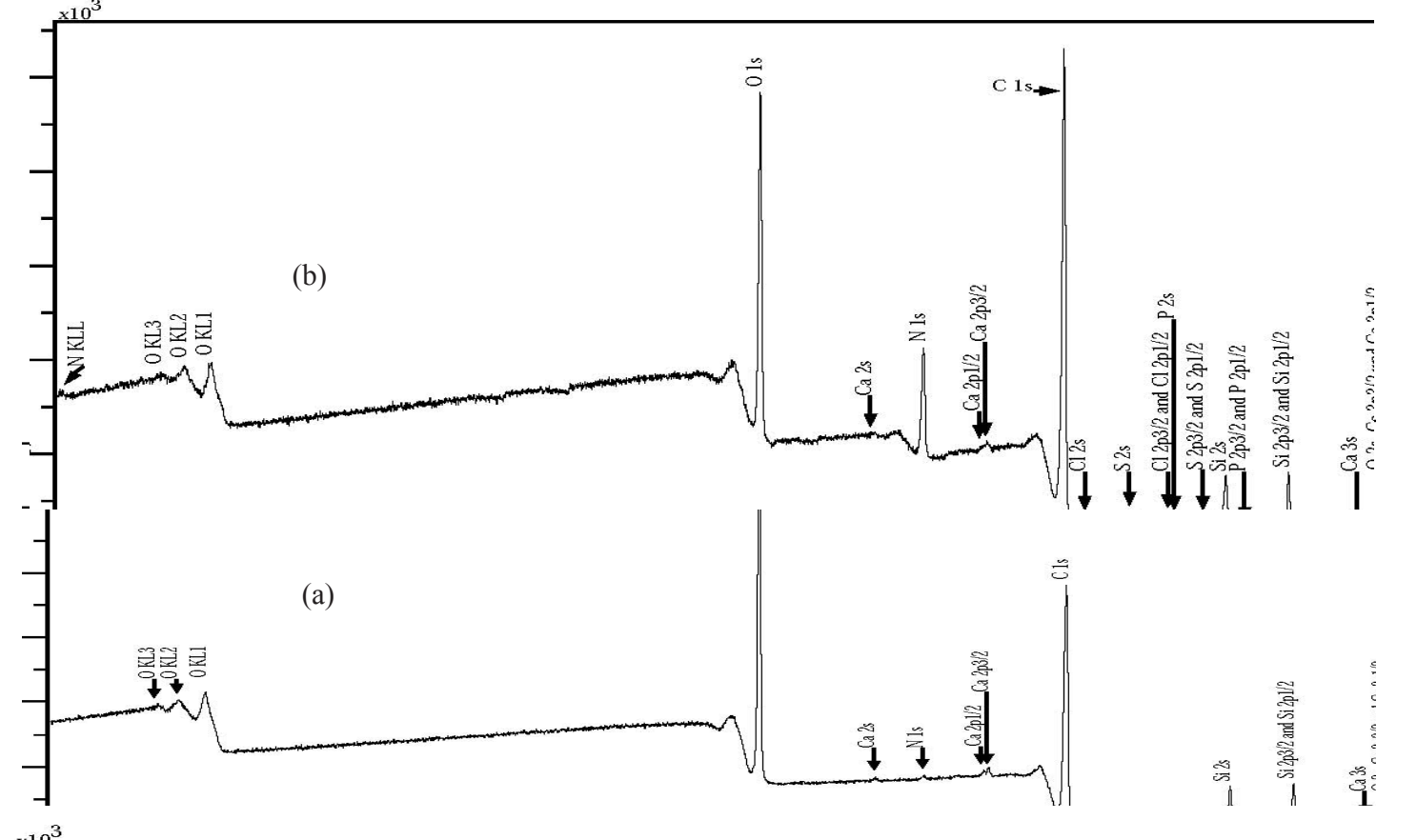

Fig. 8. Wide-scan XPS spectra of the unmodified PET/cotton fabric (a) and base hydrolyzed APTES modified PET/ cotton fabric (b). The peaks are assigned to the corresponding chemical elements.
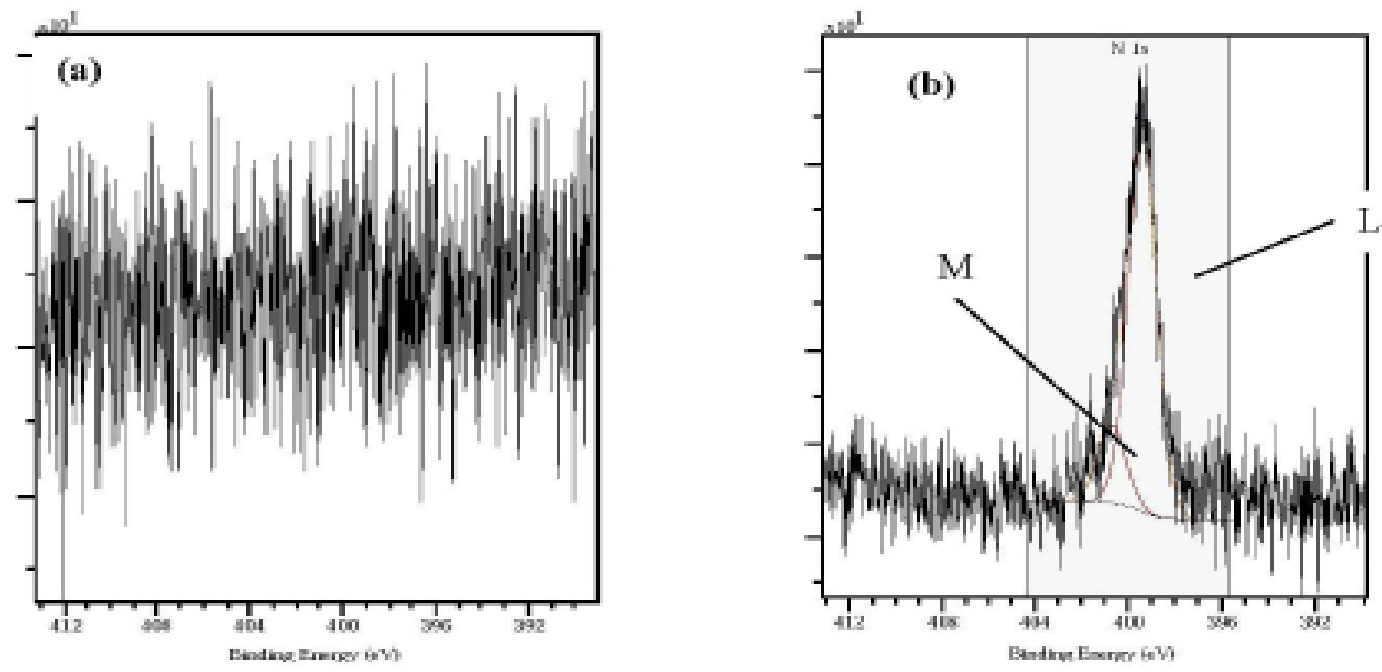

Fig. 9. High-resolution N 1s XPS spectra of the unmodified PET/cotton fabric (a) and base hydrolyzed APTES modified PET/cotton fabric (b).

TABLE 1. The elemental surface composition and atomic ratios determined by XPS for the unmodified and base hydrolyzed APTES PET/cotton fabrics.

\begin{tabular}{lccccccc}
\hline Fabric & $\mathbf{C}(\mathbf{a t .} \%)$ & $\mathbf{O}$ (at. \%) & $\mathbf{N}$ (at.\%) & Si (at. \%) & {$[\mathbf{O}]:[\mathbf{C}]$} & {$[\mathbf{N}]:[\mathbf{C}]$} & [Si]: [C] \\
\hline $\begin{array}{l}\text { Unmodified PET/cotton } \\
\text { fabrics }\end{array}$ & 60.16 & 29.32 & 0.67 & 9.49 & 0.487 & 0.011 & 0.158 \\
$\begin{array}{l}\text { Base hydrolyzed APTES } \\
\begin{array}{l}\text { PET/cotton fabrics } \\
\hline\end{array}\end{array}$ & 67.25 & 15.78 & 8.99 & 6.79 & 0.235 & 0.134 & 0.101 \\
\hline
\end{tabular}


displaying the presence of the two chemical states of nitrogen. Nitrogen species with a $\mathrm{BE}$ around $399.45 \mathrm{eV}$ attached to the phenyl ring contribute to component peak L. Component peak $\mathrm{M}$ at higher BE $400.74 \mathrm{eV}$ appears from nitrogen species attached on saturated carbon atoms, such as methylene groups.

\section{Kinetics measurements of wetting}

The effectiveness of sol-gel modification with regard to wetting properties was examined by the time-dependent contact angle measurements. Fig. 10 and 11 show the variations of waterabsorption time of water droplets of $10 \mu \mathrm{l}$ placed on the surface of the PET/cotton fabric before and after sol-gel modification under acidic or basic conditions. The variations of water-absorption time of water, shown in Fig. 10 and 11, illustrate a clear difference between the unmodified and solgel modified PET/cotton fabric either by APTES or GPTMS respectively. For the unmodified fabric, the droplet fully spreads within $14 \mathrm{~s}$ after being dropped on it. The observed hydrophilicity of unmodified fabric can be explained by the existence of polar groups such as $-\mathrm{OH}$ in the chemical structure of cellulose part of blend fabric.

On the other hand, regardless the type of alkoxysilanes, the sol-gel modification of PET/ cotton fabric resulted in the increase of water absorption time. These results show that, the hydrophobicity of the fabrics increased due to the sol-gel treatment.

The increase of water absorption time can be

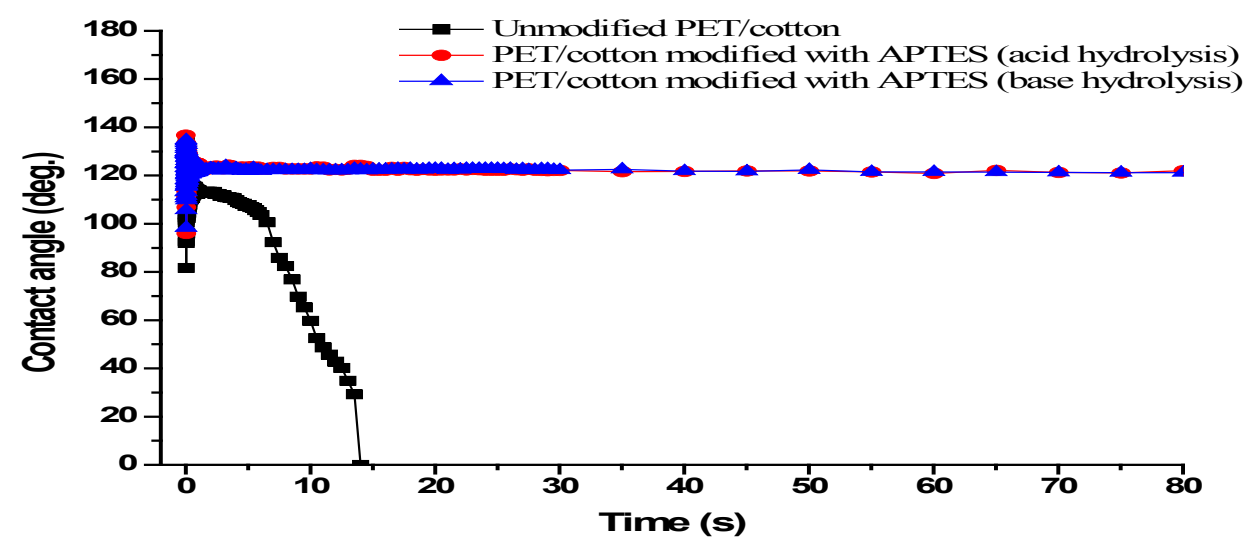

Fig. 10. Contact angles of water droplets in dependence on resting time on the unmodified PET/cotton fabric and APTES modified PET/cotton fabric under acid or base hydrolysis. The error of each measurement is $\pm 2^{\circ}$.

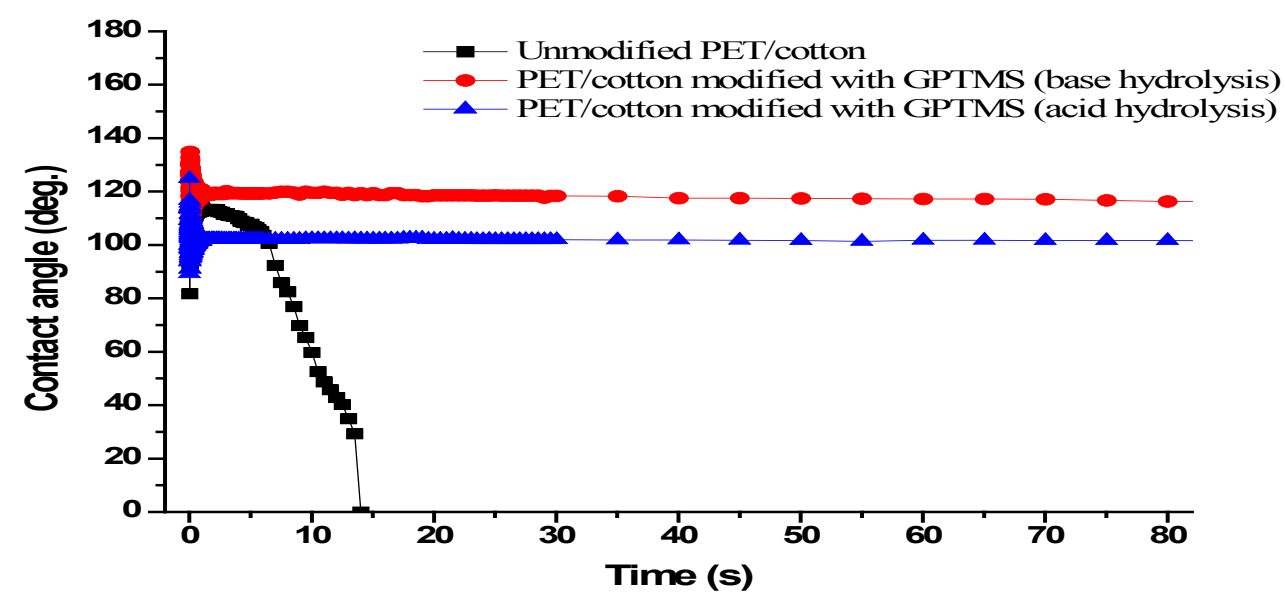

Fig. 11. Contact angles of water droplets in dependence on resting time on the unmodified PET/cotton fabric and GPTMS modified PET/cotton fabric under acid or base hydrolysis. The error of each measurement is $\pm 2^{\circ}$. 

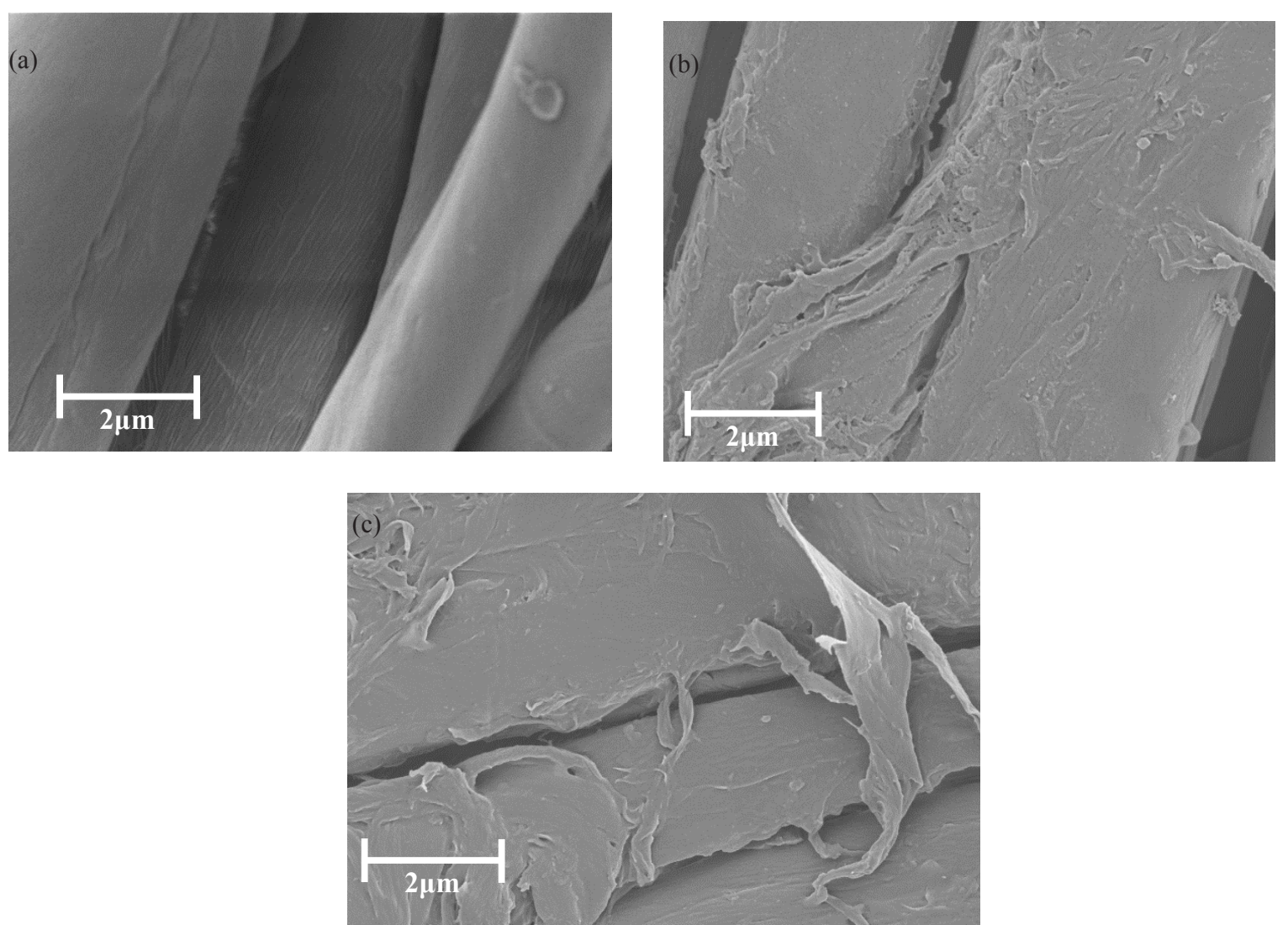

Fig. 12. SEM images of the untreated PET/cotton fabric (a), based-hydrolyzed APTES modified PET/cotton fabric (b), and based-hydrolyzed GPTMS modified PET/cotton fabric (c).

attributed to the introduction of siloxane bonds (Si-O-Si) by sol-gel treatment. These bonds enhance water repellency.

This means that sol-gel treatment switch wetting behavior of PET/cotton fabric from hydrophilic to hydrophobic.

\section{Morphology of the coating}

To follow the morphological changes after sol-gel modification step, SEM micrographs of selected base-catalysed alkoxysilanes modified PET/cotton fabric surfaces were given in Fig. 12. Fig. 12(a) illustrates SEM image of untreated PET/cotton fabric. It is obvious that PET/cotton fabric surface is relatively smooth in addition to the presence of some contaminants. It can be seen that the APTES and GPTMS layer is adhered to PET/cotton fabric surface (Fig. 12(b) and 12(c)) respectively. Moreover, we observed that the PET/cotton fabric is nearly fully covered by the APTES layer and GPTMS.

Effect of sol-gel coating on the printing behavior of PET/cotton blend fabric towards anionic dyes
In this current work, it is interesting to explore the efficiency of functional surface-modifying alkoxysilanes to modify PET/cotton blend fabric to attain solid shades using one class of dyestuffs, particularly anionic dyes. The efficiency of surface modifications has been investigated in terms of K/S results and fastness test gained from the printing experiments with different classes of anionic dyes. The color strength results of screen printed modified blend fabric with base hydrolyzed APTES and GPTMS with the C.I. Reactive Blue 5 and C.I. acid blue 80 are shown in Fig. 13.

Regardless the type of applied alkoxysilanes, the results show that the surface functionalization of PET/cotton fabric with base hydrolyzed alkoxysilanes has noticeable effect on the color strength of their prints as compared to the results of their unmodified fabrics.

In the case of using C.I. reactive blue 5, the unmodified blend fabric possessed noticeable color strength values, this is due to original substantivity of their cotton component to the 
reactive dye. It is also observed that high color strength values were obtained for modified PET/cotton fabric due to the effect acquired by functional group incorporated onto fabric such as amine group using APTES or epoxy and hydroxyl groups using GPTMS. While, in case of printing PET/cotton using C.I. acid blue 80, it is difficult to render unmodified PET/cotton fabric colored only with acid dye as shown in Fig. 13. This was expected due to the fact that, the unmodified PET/ cotton fabric does not contain reactive amine groups in their chemical composition needed for the dye/fiber reaction mechanism. Also, it is clear that the color strength values for the prints of the modified fabrics either by APTES or GPTMS are very much higher than the corresponding values of the prints of the unmodified ones.

The results displayed that PET/cotton fabric modification with either by APTES or GPTMS conducted under base hydrolysis conditions produced fabrics acquiring high $\mathrm{K} / \mathrm{S}$ values. Therefore, it may be postulated that anchoring sites introduced by alkoxysilanes surface modification are sufficient and effective in fixing dye molecules on the PET/cotton fabric surface, which can chemically bind reactive or acid dye molecules.

Finally, it was established that anchoring APTES or GPTMS layer onto PET/cotton fabric surfaces under base hydrolysis condition is an efficient strategy to improve printing behavior. This is an important step towards the substrate independent surface coloration, which becomes dependent on the top layer properties rather than chemical structure of the fibers.

\section{Fastness properties}

The color fastness to rubbing and washing of the unmodified and base hydrolyzed APTES or GPTMS modified PET/cotton blend fabrics printed with C.I. reactive blue 5 and C.I. acid blue 80 were tested (Table 2). The fastness properties were visually evaluated using a standard gray scale. Generally, the fastness properties of the modified blend fabrics range from good to excellent with C.I. reactive blue 5 and C.I. acid blue 80 as compared to the corresponding values for unmodified fabric which range from moderate to good in some cases.

\section{Conclusion}

In conclusion, the current study showed that sol-gel coating method improves the printability of PET/cotton blended fabric towards anionic dyes to produce solid shade. This new behavior induced by surface modification of PET/cotton fabric and formation of dyes bonding sites such as $-\mathrm{NH}_{2}$, epoxy and/or $-\mathrm{OH}$. These functional groups played an important role during dye-fiber interaction mechanism. Such attached groups were confirmed by streaming potential measurements and XPS. The highest values of color strength were obtained for PET/cotton modified with base hydrolyzed APTES. Also, the results demonstrated sufficient stability of prints against leaching by washing and rubbing, suggesting solgel technology formed a wash durable coating. This study may offer a simple and cost-effective method to apply durable thin coating layer bound to the surface of blended fabric, which enhances its printing behavior.

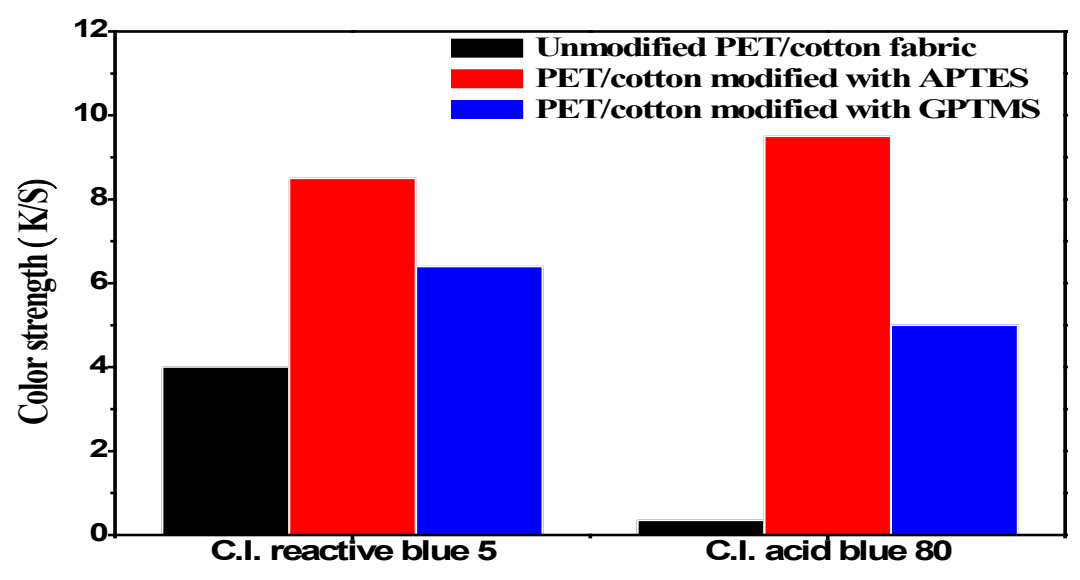

Fig. 13. Color strength of unmodified and modified PET/cotton fabrics with base hydrolyzed APTES and GPTMS using C.I. reactive blue 5 and C.I. acid blue 80.

Egypt. J. Chem. 60, No.6 (2017) 
TABLE 2. Fastness properties of unmodified and modified PET/cotton fabric with base hydrolyzed APTES or GPTMS

\begin{tabular}{cccccc}
\hline \multirow{2}{*}{ Dye } & \multirow{2}{*}{ Alkoxysilanes } & \multicolumn{2}{c}{ Rubbing fastness } & \multicolumn{2}{c}{ Washing fastness } \\
\cline { 2 - 6 } & & Dry & wet & Alt. & St. \\
\hline \multirow{3}{*}{ C.I. reactive blue 5 } & unmodified & $3-4$ & 3 & 3 & 3 \\
& APTES & $3-4$ & 3 & $4-5$ & $4-5$ \\
& GPTMS & $3-4$ & 3 & $3-4$ & $3-4$ \\
C.I. acid blue 80 & unmodified & 2 & 2 & $2-3$ & $2-3$ \\
& APTES & 4 & $3-4$ & $4-5$ & $4-5$ \\
& GPTMS & 4 & 3 & $4-5$ & $4-5$ \\
\hline
\end{tabular}

\section{Acknowledgement}

We wish to thank German Academic Exchange Service (DAAD) for the financial support. We would like also to thank Mrs. Anja Caspari, Mrs. Cornelia Krause and Mrs. Christina Elgner for their valuable contributions.

\section{References}

1. Aspland J.R., Text.Chem.Colorist, 25 (1993).

2. Chao S.M.L.Y.C., Dyes and Pigments, 37, 357371(1998).

3. Choudhury A.K.R., Textile Preparation and Dyeing. Enfield, NH: Science Publishers (2006).

4. Ruppert S., Mueller B., Banners T. and Schollmeyer, E., Text. Praxis Int., 49 (1994).

5. Mahltig B., Haufe H. and Bottcher H., Functionalisation of textiles by inorganic sol-gel coatings, Journal of Materials Chemistry, 15, 4385-4398 (2005).

6. Ferrero F. and Periolatto M., Application of fluorinated compounds to cotton fabrics via solgel, Applied Surface Science, 275, 201-207, 6/15/ (2013).

7. Wei, Q., Surface Modification of Textiles: Woodhead Publishing Limited and CRC Press LLC (2009).

8. Caldara M., Colleoni C., Guido E., Re V. and Rosace G., Development of a textileoptoelectronic $\mathrm{pH}$ meter based on hybrid xerogel doped with Methyl Red, Sensors and Actuators B: Chemical, 171-172, 1013-1021, 8// (2012).

9. Capeletti L.B., Dos Santos J.H.Z., Moncada E., Da Rocha Z.N. and Pepe I.M., Encapsulated alizarin red species: The role of the sol-gel route on the interaction with silica matrix, Powder Technology, 237, 117-124, 3// (2013).
10. Barbé C., Kong L., Finnie K., Calleja S., Hanna J. and Drabarek E., Sol-gel matrices for controlled release: from macro to nano using emulsion polymerisation, Journal of Sol-Gel Science and Technology, 46, 393-409, 2008/06/01 (2008).

11. Satoh K., Nakazumi H. and Morita M., Novel fluorinated inorganic-organic finishing materials for nylon carpeting, Textile Research Journal, 74, 1079-1084, December 1, 2004 (2004).

12. Černe L. and Simončič B., Influence of repellent finishing on the surface free energy of cellulosic textile substrates, Textile Research Journal, 74, 426-432, May 1, 2004.

13. Chen D., Tan L., Liu H., Hu, J., Li Y. and Tang F., Fabricating superhydrophilic wool fabrics, Langmuir, 26, 4675-4679, 2010/04/06 (2009).

14. Gao Q., Zhu Q., Guo Y. and Yang C.Q., Formation of highly hydrophobic surfaces on cotton and polyester fabrics using silica sol nanoparticles and nonfluorinated alkylsilane, Industrial \& Engineering Chemistry Research, 48, 9797-9803, 2009/11/18 (2009).

15. Textor T. B. T. and Schollmeyer E., J. Ind. Text, 32, 279-289 (2003).

16. Daoud J. H. X. W. A., J. Sol-Gel Sci. Technol., 29, 25-29(2004).

17. McIntyre J. E., Synthetic Fibres: Nylon, Polyester, Acrylic, Polyolefin: Woodhead Publishing Limited in association with The Textile Institute (2005).

18. Zou Y., Reddy N. and Yang Y., Reusing polyester/ cotton blend fabrics for composites, Composites Part B: Engineering, 42, 763-770, 6// (2011).

19. Youssef Y. A., Ahmed N. S. E., Mousa A. A. and El-Shishtawy R. M., Alkaline dyeing of polyester and polyester/cotton blend fabrics using sodium edetate, Journal of Applied Polymer Science, 108, 342-350 (2008).

Egypt. J. Chem. 60, No.6 (2017) 
20. Oktem T., Seventekin N., Ayhan H. and Piskin E., Indian J. Fibre Text Res, 27, 161-165, (2002).

21. Ristić N., Jovančić P., Canal C. and Jocić D., Onebath one-dye class dyeing of PES/cotton blends after corona and chitosan treatment, Fibers and Polymers, 10, 466-475, 2009/08/01 (2009).

22. Hinks D., Rashad M. , and El-Shafei, A., Towards a universal dye class for printing on multiple substrates, Coloration Technology, 119, 70-75, (2003).

23. Beamson G. and Briggs D., High Resolution XPS of Organic Polymers: Wiley (1992).

24. Shirley D.A., High-resolution X-ray photoemission spectrum of the valence bands of gold, Physical Review B, 5, 4709, (1972).

25. Delgado A., González-Caballero F., Hunter R., Koopal L. and Lyklema J., Measurement and interpretation of electrokinetic phenomena (IUPAC technical report). Pure and Applied Chemistry, 77, 1753-1805 (2005).

26. Dutschk V., Sabbatovskiy K. G., Stolz M., Grundke
K. and Rudoy V. M., Unusual wetting dynamics of aqueous surfactant solutions on polymer surfaces, Journal of Colloid and Interface Science, 267, 456-462 (2003).

27. Miles L.W. and Leslie W., Textile Printing. Revised, ed: Society of Dyers and Colourists, (2003).

28. Alvarez J. and Lipp-Symonowicz B., Examination of the absorption properties of various fibres in relation to UV radiation, Autex Research Journal, 3, 72-77(2003).

29. Amirshahi S. and Pailthorpe M., Reduction of quenching effects of fluorescent whitening agents by blending, Dyes and Pigments, 26, 121-128, (1994).

30. Stern O., Theory of the electrical double layer. (In German.), Electrochemistry, 30, 508-516 (1924).

(Received 29/8/2017; accepted 3/10/2017)

\section{طريقة سول-جيل الاصطناعية لتحسين تفاعل قماش البوليستر/قطن المخلوط مع الصبغات

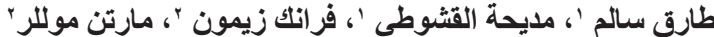

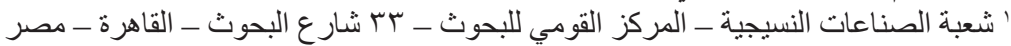

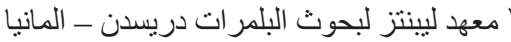

اختلاف الطبيعة الكيميائية للألياف المصنوع منها قماش البوليستر/قطن المخلوط يعوق الحصول علي ظل الحل لوني

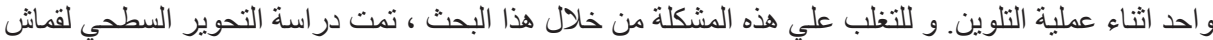

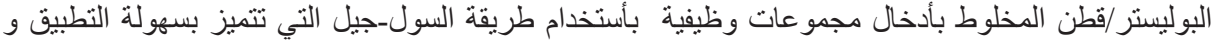

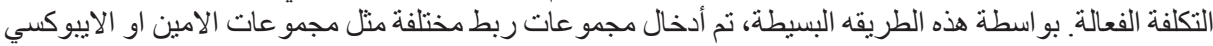

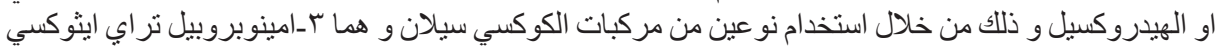

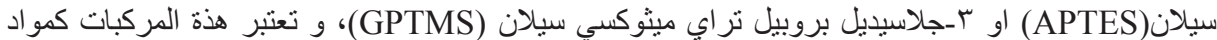

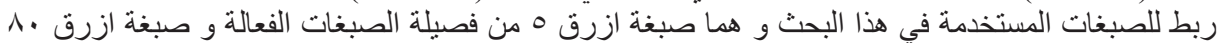

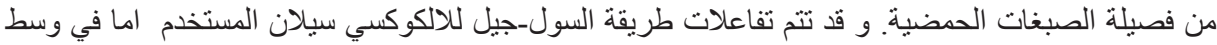

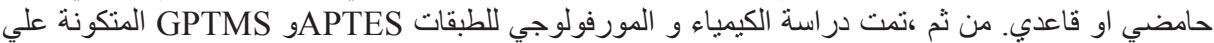

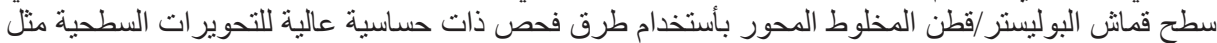

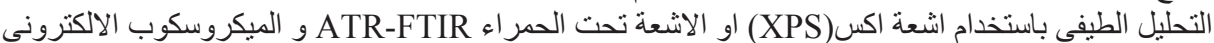

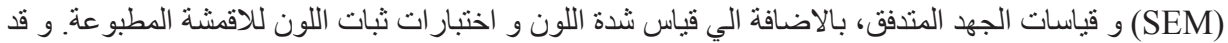

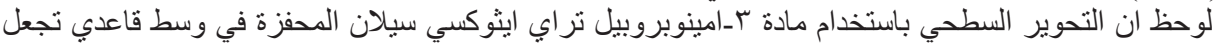

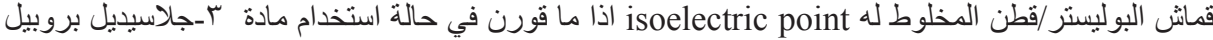

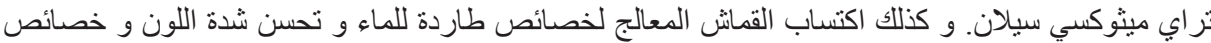

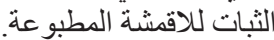

